International Journal of Difference Equations (IJDE).

ISSN 0973-6069, Volume 15, Number 1, (2020). 107-126

(C) Research India Publications

https://dx.doi.org/10.37622/IJDE/15.1.2020.107-126

\title{
Oscillation and Asymptotic Behavior of Second-Order Neutral Delay Difference Equations with Variable Delays
}

\author{
C. R. Behera ${ }^{1}$ and R. N. Rath ${ }^{* 2}$ \\ ${ }^{1}$ Department of Mathematics, S. I. T., Bhubaneswar, 751024, Odisha, India.
}

${ }^{2}$ Ex. Professor (VSSUT Burla, 768018) and Retired Principal, Khallikote Autonomous College, Berhampur, 760001, Odisha, India.

\begin{abstract}
In this article, sufficient conditions are obtained for the oscillation of all solutions of the second-order neutral delay difference equation

$$
\Delta^{2}\left(y_{n}-\sum_{j=1}^{k} p_{n}^{\{j\}} y_{n-m_{j}}\right)+v_{n} G\left(y_{\sigma(n)}\right)-u_{n} H\left(y_{\alpha(n)}\right)=f_{n},
$$

where $\Delta x_{n}=x_{n+1}-x_{n}$. This paper, improve and generalize some known results. Further the results are illustrated by examples, to which no result in the literature could be applied.
\end{abstract}

Key Words and Phrases: Oscillatory solution; non oscillatory solution; asymptotic behaviour, difference equation.

2000 Mathematics Subject Classification: 39A10, 39A12.

\footnotetext{
${ }^{*}$ Corresponding author
} 


\section{INTRODUCTION}

In this article, we investigate to find sufficient conditions for the oscillation and asymptotic behavior of solutions of a second order neutral delay difference equation (NDDE in short)

$$
\Delta^{2}\left(y_{n}-\sum_{j=1}^{k} p_{n}^{\{j\}} y_{n-m_{j}}\right)+v_{n} G\left(y_{\sigma(n)}\right)-u_{n} H\left(y_{\alpha(n)}\right)=f_{n}
$$

where $\Delta x_{n}=x_{n+1}-x_{n}$, and $\Delta^{2} x_{n}=\Delta\left(\Delta x_{n}\right)$. We assume that $v_{n}>0, u_{n} \geq 0$ and $f_{n}$ are real sequences; $m_{1}, m_{2}, \ldots, m_{k}$ are non-negative integers; $\alpha(n)$ and $\sigma(n)$ are sequences of integers which are less than $n$ and tend to $\infty$ as $n \rightarrow \infty$; and the functions $G$ and $H$ belong $C(\mathbb{R}, \mathbb{R})$. We assume $x H(x)>0$, so we can study (1.1) with positive and with negative coefficients. Different conditions on the real bounded sequences $\left\{p_{n}^{\{j\}}\right\}_{j=1}^{k}$ are considered. The results in this paper also hold when $u_{n} \equiv 0$, $f_{n} \equiv 0$ and $G\left(u_{n}\right) \equiv u_{n}$ in (1.1).Some of the following assumptions are to be used later in this article.

(G0) $\liminf \operatorname{in\rightarrow \infty }_{n \rightarrow \infty} G\left(x_{n}\right)>0$ if $\liminf _{n \rightarrow \infty} x_{n}>0$ and $\limsup _{n \rightarrow \infty} G\left(x_{n}\right)<0$ if $\limsup _{n \rightarrow \infty} x_{n}<0$.

(G1) $x G(x)>0$ for $x \neq 0$.

(G2) $v_{n}>0, \sum_{n=n_{0}}^{\infty} v_{n}=\infty$.

(G3) There exists a bounded sequence $\left\{F_{n}\right\}$ such that $\Delta^{2} F_{n}=f_{n}$.

(G4) The sequence $F_{n}$ in (G3) satisfies $\lim _{n \rightarrow \infty} F_{n}=0$.

(G5) $\sum_{n=n_{0}}^{\infty} n u_{n}<\infty$.

(G6) $H$ is bounded.

(G7) $\sum_{n=n_{0}}^{\infty} n v_{n}=\infty$.

(G8) $\sum v_{n j}=\infty$.where $v_{n j}$ is any subsequence of $v_{n}$.

A great deal of work is done and much more is to come by researchers and scientists on the oscillatory and asymptotic behavior of neutral delay difference equations due to its various application (see[4]) in the fields of science and technology. However, the study of second order NDDEs (see [2, 7, 12, 13, 14]) with coefficient function changing sign [15] seems to be very rare. Particularly, study of second order NDDE (1.1) with multiple delays under $\Delta^{2}$ and the coefficient function changing sign is yet to be taken. 
The results obtained for the oscillation of (1.1) is applied to obtain the sufficient conditions for oscillatory and asymptotic behavior of solutions of the neutral difference equation

$$
\Delta^{2}\left[y_{n}-\sum_{i=1}^{k} p_{n}^{\{i\}} y_{n-m_{i}}\right]+q_{n} G\left(y_{\sigma(n)}\right)=f_{n}
$$

where $q_{n}$ changes sign. We observe that the condition that "for $|u|>0$ there exists $\delta>0$ such that $|G(u)| \geq \delta$ "was assumed in a recent publication [2] for the study of qualitative behavior of (1.2), does not hold for sublinear $G$, i.e; when $G(x)=x^{1 / 3}$. Further, note that, the results in [2] does not hold for the general case, when $q_{n}$ in (1.2) changes sign. However, the results of this article complements the work [2] and the results hold for the cases when $G$ is linear, super linear or sublinear and also for the general case that, $q_{n}$ changing sign in (1.2). Further, these results improve and generalize the work in [13]which deals with the study of the second order non linear homogeneous neutral equation

$$
\Delta^{2}\left(y_{n}-p_{n} y_{n-m}\right)+v_{n} G\left(y_{n-\sigma}\right)-u_{n} H\left(y_{n-r}\right)=0
$$

Let $n_{1}$ be a fixed non-negative integer and

$$
n_{0}=\min \left\{n_{1}-\max _{1 \leq j \leq k} m_{j}, \min _{n \geq n_{1}} \sigma(n), \min _{n \geq n_{1}} \alpha(n)\right\} .
$$

By a solution of (1.1) we mean a sequence of real numbers $\left\{y_{n}\right\}$ defined for $n \geq n_{0}$ that satisfies (1.1) for $n \geq n_{1}$. If a set of values

$$
y_{n}=a_{n} \text { for } n_{0} \leq n \leq n_{1}+1 \text {, }
$$

is given, then (1.1) defines an equation in which we can solve for $y_{n_{1}+2}$ explicitly, and we obtain a unique solution of (1.1) satisfying the given initial condition (1.4).

A solution $\left\{y_{n}\right\}$ of (1.1) is eventually positive if $y_{n}>0$ for all $n$, large enough, and eventually negative if $y_{n}<0$ for all $n$, large enough. A nontrivial solution is oscillatory if it is neither eventually positive nor eventually negative.

The arrangement of the contents of this article is as follows. In section $\S 1$, brief review of literature, the motivation behind the work and the assumptions required, are stated. In $\S 2$, sufficient conditions are found for every solution of (1.1) to be oscillatory or tending to zero as $n \rightarrow \infty$. In $\S 3$, we find sufficient conditions for oscillatory and asymptotic behaviour of solutions of the neutral difference equation (1.2), where the coefficient function $q_{n}$ changes sign. Finally in $\S 4$ concluding remark is given. 


\section{SUFFICIENT CONDITION}

In this section, we present few lemmas from $[1,2,9]$ that would be useful for our results in the sequel.

Lemma 2.1. [2, Lemma 2.12] Assume $y_{n}>0$ for $n \geq n_{0}$ with $\liminf _{n \rightarrow \infty} y_{n}=0$. Suppose that

$$
z_{n}=y_{n}-\sum_{j=1}^{k} p_{n}^{\{j\}} y_{n-m_{j}}
$$

Further, assume that $\lim _{n \rightarrow \infty} z_{n}=\delta$ exists finitely. Then

(a) If $p_{n}^{\{j\}} \geq 0$ then $\delta \leq 0$ and $p_{n}^{\{j\}} \leq 0$ then $\delta \geq 0$.

(b) Further, suppose that $y_{n}$ is bounded and the bounded sequences $p_{n}^{\{j\}}, j=1,2, \ldots, k$, satisfy one of the following four conditions.

$$
\begin{gathered}
b_{j} \geq p_{n}^{\{j\}} \geq 0 \quad \text { for every } \quad j=1,2, \ldots k \quad \text { and } \quad \sum_{j=1}^{k} \lim \sup p_{n}^{\{j\}}<b<1 . \\
-b_{j} \leq p_{n}^{\{j\}} \leq 0 \quad \text { for every } \quad j=1,2, \ldots k \text { and } \quad \sum_{j=1}^{k} \liminf p_{n}^{\{j\}}>-b>-1 .
\end{gathered}
$$

$-b_{j} \leq p_{n}^{\{j\}} \leq 0 \quad$ for every $\quad j=1,2, \ldots k \quad$ and there exists $i \in\{1,2,3, \ldots, k\}$ such that

$$
\limsup p_{n}^{\{i\}}-\sum_{j \neq i} \liminf p_{n}^{\{j\}}<-1 .
$$

$b_{j} \geq p_{n}^{\{j\}} \geq 0 \quad$ for every $\quad j=1,2, \ldots k \quad$ and there exists $i \in\{1,2,3, \ldots, k\}$ such that

$$
\liminf p_{n}^{\{i\}}-\sum_{j \neq i} \lim \sup p_{n}^{\{j\}}>1 .
$$

In the above $b_{1}, b_{2}, \ldots, b_{k}$ and $b$ are scalars such that $b_{1}+b_{2}+\ldots+b_{k}=b$. Then $\delta=0$ and $\lim _{n \rightarrow \infty} y_{n}=0$.

Lemma 2.2. [1] Let $z_{n}$ be a real valued function defined for $n \in N\left(n_{0}\right)=\left\{n_{0}, n_{0}+\right.$ $1, \ldots\}, n_{0} \geq 0$ and $z_{n}>0$ with $\Delta^{m} z_{n}$ of constant sign on $N\left(n_{0}\right)$ and not identically zero.Then there exists an integer $p, 0 \leq p \leq m-1$, with $m+p$ odd for $\Delta^{m} z_{n} \leq 0$ and $(m+p)$ even for $\Delta^{m} z_{n} \geq 0$, such that

$$
\Delta^{i} z_{n}>0 \text { for } n \geq n_{0}, 0 \leq i \leq p
$$

and

$$
(-1)^{p+i} \Delta^{i} z_{n}>0, \text { for } n \geq n_{0}, p+1 \leq i \leq m-1 .
$$


Remark 2.3. From the above lemma, for $m=2$, if $\Delta^{2} z_{n} \leq 0$ and $z_{n} \geq 0$ then $p=1$ and $\Delta z_{n}>0$.

Lemma 2.4. [9] Let $m \in \mathbb{N}$ and $x(n)$ be a non oscillatory sequence which is positive for large $n$. If there exists an integer $p \in\{0,1, \ldots, m-1\}$ such that $\Delta^{p} w(\infty)$ exits (finite) and $\Delta^{i} w(\infty)=0$ for all $i \in\{p+1, \ldots, m-1\}$. Then

$$
\Delta^{m} w(n)=-x(n)
$$

implies

$$
\Delta^{p} w(n)=\Delta^{p} w(\infty)+\frac{(-1)^{m-p-1}}{(m-p-1) !} \sum_{i=n}^{\infty}(i+m-p-1-n)^{(m-p-1)} x(i),
$$

for all sufficiently large $n$.

Theorem 2.5. Suppose that (G1), (G3)-(G5),(G7) hold. Assume that $p_{n}^{\{j\}}$ for $j=$ $1,2,3, \ldots, k$ satisfies one of the conditions $(2.2)-(2.5)$. Then every bounded solution of (1.1) oscillates or tends to zero as $n \rightarrow \infty$.

Proof. Assume $y_{n}$ is a bounded and eventually positive solution of (1.1). Then there exists a $n_{0}$ such that for $n \geq n_{0}: y_{n}, y_{\alpha(n)}, y_{\sigma(n)}, y_{n-m_{i}}$ for each $i$ are positive.

Define

$$
c_{n}=-\sum_{s=n}^{\infty}(s-n+1) u_{s} H\left(y_{\alpha(s)}\right) \quad \text { for } n \geq n_{0} .
$$

By assumptions (G5) and (G6), the above summation converges, thus $c_{n}$ is a well defined real-valued sequence, and

$$
\lim _{n \rightarrow \infty} c_{n}=0
$$

Note that the 2nd order difference of $c_{n}$ is $\Delta^{2} c_{n}=-u_{n} H\left(y_{\alpha(n)}\right)$. For simplicity of notation, we define $z_{n}$ as in (2.1) and

$$
w_{n}=z_{n}+c_{n}-F_{n},
$$

where $\Delta^{2} F_{n}=f_{n}$. Since $v_{n}>0$, then from (2.10), (G1) and (1.1) it follows that

$$
\Delta^{2} w_{n}=-v_{n} G\left(y_{\sigma(n)}\right) \leq 0
$$

Then it follows from (2.11) that $w_{n}, \Delta w$ are monotonic and of constant sign on some interval $\left[n_{1}, \infty\right)$. As $y_{n}$ is bounded, then $z_{n}$ and $w_{n}$ are bounded. Let $\lambda:=\lim _{n \rightarrow \infty} w_{n}$ 
which exists as a finite number because $w_{n}$ is monotonic and bounded. Applying Lemma 2.4 to (2.11) i.e; twice summation on (2.11), we obtain

$$
w_{n}=\lambda-\sum_{s=n}^{\infty}(s-n+1) v_{s} G\left(y_{\sigma(s)}\right) .
$$

Since $w_{n}$ is bounded, the above summation is convergent. That is

$$
\sum_{s=n}^{\infty}(s-n+1) v_{s} G\left(y_{\sigma(s)}\right)<\infty .
$$

This in turn, by (G7), implies $\liminf _{s \rightarrow \infty} G\left(y_{\sigma(s)}\right)=0$. As $G(x) \neq 0$ for $x \neq 0$, $\liminf _{s \rightarrow \infty} y_{\sigma(s)}=0$, other wise there exists a positive scalar $\alpha$ and a large integer $n_{2} \geq n_{1}$ such that $y_{\sigma(n)} \geq \alpha>0, n \geq n_{2}$. Boundedness of $y_{n}$ gives us an upper bound $\beta$ for $y_{\sigma(n)}$. Since $G$ is continuous, the term $G\left(y_{\sigma(n)}\right)$ has a positive lower bound $\gamma$, a contradiction. Thus, $\liminf _{s \rightarrow \infty} y_{\sigma(s)}=0$. Because $\lim _{n \rightarrow \infty} \sigma(n)=\infty$, $\liminf _{n \rightarrow \infty} y_{n}=0$. Since $\lim _{n \rightarrow \infty} w_{n}$ exists, $c_{n}, F_{n}$ approach zero, and each $p_{n}^{\{j\}}, j=$ $1,2, \ldots, k$ are bounded, it follows that $\lim _{n \rightarrow \infty} z_{n}$ exists as a finite number. Applying Lemma 2.1 we prove $\lim _{n \rightarrow \infty} y_{n}=0$.

If $y_{n}$ is an eventually negative solution of (1.1) for large $n$ then we set $x_{n}=-y_{n}$ to obtain $x_{n}>0$ and then (1.1) reduces to

$$
\Delta^{2}\left(x_{n}-\sum_{j=1}^{k} p_{n}^{\{j\}} x_{n-m_{j}}\right)+v_{n} \tilde{G}\left(x_{\sigma(n)}\right)-u_{n} \tilde{H}\left(x_{\alpha(n)}\right)=\tilde{f}_{n},
$$

where

$$
\tilde{f}_{n}=-f_{n}, \quad \tilde{G}(v)=-G(-v) \quad \tilde{H}(v)=-H(-v) .
$$

Further,

$$
\tilde{F}_{n}=-F_{n} \text { implies } \Delta^{2}\left(\tilde{F}_{n}\right)=\tilde{f}_{n} .
$$

In view of the above facts, it can be easily verified that $\tilde{G}, \tilde{H}$ and $\tilde{F}$ satisfy the corresponding conditions satisfied by the functions $G, H$ and $F$ in the theorem. Proceeding with (2.12) as in the proof for the case $y_{n}>0$, we may complete the proof of the theorem.

Remark 2.6. The above theorem 2.5 generalizes [2][Theorem 3.4] and extends[10][ Theorem 6].

Theorem 2.7. Suppose that (G0)-(G6) hold. Assume that there exists a positive constant $b$ such that the sequence $p_{n}^{\{j\}}: j=1,2, \ldots, k$ satisfies the condition (2.3). Then every solution of (1.1) oscillates or tends to zero as $n \rightarrow \infty$. 
Proof. Let $y_{n}$ be a solution of (1.1), which is eventually positive for $n \geq n_{0}$. Then proceeding as in Theorem 2.5, we define $z_{n}, c_{n}$ and $w_{n}$ by (2.1),(2.8) and (2.10) respectively to obtain (2.11). By $(\mathrm{G} 2), \Delta^{2} w_{n}$ is not identically zero in any interval $\left[n_{0}, \infty\right)$. It follows that there exists $n_{1} \geq n_{0}$ such that $w_{n}, \Delta w_{n}$ are monotonic and of constant sign on $\left[n_{1}, \infty\right)$. However, we do not know yet that $w_{n}>0$.

If possible let $y_{n}$ be unbounded, then, there exists an increasing sequence $\left\{a_{j}\right\}$ such that

$$
\lim _{j \rightarrow \infty} a_{j}=\infty, \quad \lim _{j \rightarrow \infty} y_{a_{j}}=\infty, \quad \text { with } y_{a_{j}}=\max _{n_{1} \leq s \leq a_{j}} y_{s} .
$$

By (2.9), for each $\epsilon>0$, there exists $N_{0}$ such that

$$
c_{a_{j}}<\epsilon \quad \text { for } j \geq N_{0} \text {. }
$$

Since $\sigma(n)$, each $n-m_{i}$ approach $\infty$ as $n \rightarrow \infty$, there exists $N_{1} \geq N_{0}$ such that: $\sigma\left(a_{j}\right)$, each $a_{j}-m_{i}>n_{1}$ for $j \geq N_{1}$. By (G3), there is an upper bound $\eta$ for $\left|F_{n}\right|$. Using that $y_{n}>0$, the definition of $\left\{a_{j}\right\}$, and that $n-m_{i} \leq n$, we have: by (2.3),

$$
\begin{gathered}
w_{a_{j}}=y_{a_{j}}-\sum_{i=1}^{k} p_{a_{j}}^{\{i\}} y_{a_{j}-m_{i}}+c_{a_{j}}-F_{a_{j}} \\
\geq(1-b) y_{a_{j}}-\epsilon-\eta, \quad j \geq N_{1} .
\end{gathered}
$$

Taking limits in the inequality above, we have $\lim _{j \rightarrow \infty} w_{a_{j}}=\infty$. Since $w_{n}, \Delta w_{n}$ are monotonic and of constant sign, it follows that $w_{n}>0$ and $\Delta w_{n}>0$. As $\Delta^{2} w_{n} \leq 0$ and $w_{n}>0$ then by lemma 2.2and remark2.3 one may have $\Delta w_{n}>0$ for $n \geq n_{1}$. Next it is to be shown that $y_{n}$ is bounded below by a positive constant, which will be used for bounding the $G$ term from below. From (2.3) and that $w_{n}$ is positive and increasing, it follows that

$$
\begin{aligned}
\left(1-\sum_{i=1}^{k} b_{i}\right) w_{n} & \leq w_{n}-\sum_{i=1}^{k} b_{i} w_{n-m_{i}} \leq w_{n}+\sum_{i=1}^{k} p_{n}^{\{i\}} w_{n-m_{i}} \\
& =y_{n}-\sum_{i=1}^{k} p_{n}^{\{i\}} y_{n-m_{i}}+c_{n}-F_{n} \\
& +\sum_{i=1}^{k} p_{n}^{\{i\}}\left[y_{n-m_{i}}-\left[\sum_{j=1}^{k} p_{n-m_{i}}^{\{j\}} y_{n-m_{i}-m_{j}}\right]+c_{n-m_{i}}-F_{n-m_{i}}\right] \\
& \leq y_{n}+c_{n}-F_{n}+\sum_{i=1}^{k} p_{n}^{\{i\}}\left[c_{n-m_{i}}-F_{n-m_{i}}\right]
\end{aligned}
$$


Note that each $p_{n}^{\{i\}}<0$ and $p_{n-m_{j}}^{\{i\}}<0, i=1,2, \ldots, k, j=1,2, \ldots, k$ and $y_{n}>0$ in the inequality above. This implies

$$
\left(1-\sum_{i=1}^{k} b_{i}\right) w_{n} \leq y_{n}+\left|c_{n}\right|+\left|F_{n}\right|+\sum_{i=1}^{k}\left|p_{n}^{\{i\}}\right|\left[\left|c_{n-m_{i}}\right|+\left|F_{n-m_{i}}\right|\right] .
$$

Then it follows that

$$
\left(1-\sum_{i=1}^{k} b_{i}\right) w_{n} \leq y_{n}+\epsilon+\eta+\sum_{i=1}^{k} b_{i}(\epsilon+\eta) \text { for } n \geq n_{1} .
$$

Since $\lim _{n \rightarrow \infty} w_{n}=\infty$, it follows that $\lim _{n \rightarrow \infty} y_{n}=\infty$. Then there exists $n_{2} \geq n_{1}$ such that for $n \geq n_{2}: y_{n}, y_{\alpha(n)}, y_{\sigma(n)}, y_{n-m_{i}}$ for each $i$ are bounded below by a positive constant. Due to (G0), (G1) and continuity of $G$, we find that, for $s \geq n_{2}, G\left(y_{\sigma(n)}\right)$ is bounded below by a positive constant $\beta$. Taking summation on (2.11),

$$
\Delta w_{n}=\Delta w_{n_{2}}+\sum_{s=n_{2}}^{\infty}-v_{s} G\left(y_{\sigma(s)}\right) \leq \Delta w_{n_{2}}-\beta \sum_{s=n_{2}}^{\infty} v_{s} .
$$

Note that by (G2), the right-hand side approaches $-\infty$, while the left-hand side is positive. This contradiction implies that the solution can not be unbounded and eventually positive. Hence $y_{n}$ is bounded. Since (G2) implies (G7) then we proceed as in Theorem 2.5 to prove $\lim _{n \rightarrow \infty} y_{n}=0$. If $y_{n}$ is an eventually negative solution of (1.1) for large $n$ then we set $x_{n}=-y_{n}$ to obtain $x_{n}>0$ and then (1.1) reduces to

$$
\Delta^{2}\left(x_{n}-\sum_{j=1}^{k} p_{n}^{\{j\}} x_{n-m_{j}}\right)+v_{n} \tilde{G}\left(x_{\sigma(n)}\right)-u_{n} \tilde{H}\left(x_{\alpha(n)}\right)=\tilde{f}_{n},
$$

Further, note that (2.13) and (2.14) hold. Then we may proceed with (2.15) as in the proof for the case $y_{n}>0$ and complete the proof of the theorem.

Theorem 2.8. Suppose that (G0)-(G6) hold. Assume that there exists a positive constant $b$ such that $p_{n}^{\{j\}}: j=1,2, \ldots, k$ satisfies the condition (2.2). Then every solution of (1.1) oscillates or tends to zero as $n \rightarrow \infty$.

Proof. By contradiction assume $y_{n}$ to be an eventually positive solution of (1.1), which does not tend to zero as $n \rightarrow \infty$. Then there exists a $n_{0}$ such that for $n \geq n_{0}$ : $y_{n}, y_{\alpha(n)}, y_{\sigma(n)}$ are positive and $\lim _{\sup _{n \rightarrow \infty}} y_{n}>0$. Define $c_{n}, z_{n}$ and $w_{n}$ by (2.8),(2.1) and (2.10) respectively to obtain (2.11). By (G2), $\Delta^{2} w_{n}$ is not identically zero in any interval $\left[n_{1}, \infty\right)$. Then from Lemma 2.2, it follows that $w_{n}, \Delta w_{n}$ are monotonic and of 
constant sign on some interval $\left[n_{1}, \infty\right)$. We do not know that $w_{n}>0$ yet. Since (2.2) holds,

$$
w_{n} \geq y_{n}-\sum_{i=1}^{k} b_{i} y_{n-m_{i}}+c_{n}-F_{n} .
$$

Taking the limit superior, using that $w_{n}$ is monotonic and that $c_{n}$ and $F_{n}$ converge to zero, we have

$$
\lambda=\lim _{n \rightarrow \infty} w_{n} \geq\left(1-\sum_{i=1}^{k} b_{i}\right) \limsup _{n \rightarrow \infty} y_{n}>0 .
$$

Then $w_{n}$ is positive for $n$ large enough. By [6, Lemma 5.2.1], $\Delta^{2} w_{n} \leq 0$ and $w_{n}>0$ imply the existence of $n_{1}$ such that $\Delta w_{n}>0$ for $n \geq n_{1}$. Next we show that $\liminf _{n \rightarrow \infty} y_{n}>0$, which will be used for bounding $G\left(y_{\sigma(n)}\right)$ from below by a positive constant. Using that $0 \leq p_{n}^{\{j\}}, j=1,2, \ldots, k$ and $y_{n}>0$, we have

$$
w_{n} \leq y_{n}+c_{n}-F_{n} .
$$

Taking the limit inferior, using that $w_{n}$ is monotonic and that $c_{n}$ and $F_{n}$ approach zero, we have

$$
0<\lambda=\lim _{n \rightarrow \infty} w_{n} \leq \liminf _{n \rightarrow \infty} y_{n} .
$$

Then there exists a $n_{2} \geq n_{1}$ such that for $n \geq n_{2}: y_{n}, y_{\alpha(n)}, y_{\sigma(n)}$, are bounded below by a positive constant. Using (G0), (G1) and continuity of $G$, we find for $s \geq n_{2}, G\left(y_{\sigma(n)}\right)$ is bounded below by a positive constant $\beta$. Taking summation on (2.11),

$$
\Delta w_{n}=\Delta w_{n_{2}}+\sum_{s=n_{2}}^{\infty}-v_{s} G\left(y_{\sigma(s)}\right) \leq \Delta w_{n_{2}}-\beta \sum_{s=n_{2}}^{\infty} v_{s} .
$$

Note that by (G2), the right-hand side approaches $-\infty$, while the left-hand side is positive. This contradiction implies that the solution can not be eventually positive without approaching zero.

If $y_{n}$ is eventually negative and does not tend to zero as $n \rightarrow \infty$, then proceeding as in the above, we complete the proof of the theorem.

Remark 2.9. The above two Theorems 2.7 and 2.8 extend [10][Theorems 1 and 2] and generalize [2][Theorem 3.1].

Corollary 2.10. If $p_{n}^{\{j\}}$ satisfy (2.3) or (2.2) and (GO) -(G6) hold then every unbounded solution of (1.1) oscillates.

Theorem 2.11. Assume (G0), (G1, (G3)-(G6). Further, assume that $p_{n}^{\{j\}}, j=$ $1,2, \ldots, k$ satisfy

$$
-b \leq p_{n}^{\{j\}} \leq 0 \quad \text { for each } j
$$


Let $\sigma\left(n-m_{j}\right)=\sigma(n)-m_{j}$ for $j=1,2, \ldots, k$. Suppose that

$$
\sum_{n=n_{0}}^{\infty} v_{n}^{*}=\infty ; \quad \text { where } v_{n}^{*}=\min \left\{v_{n}, v_{n-m_{1}}, \ldots, v_{n-m_{k}}\right\} .
$$

Further, suppose that there exists a positive constant $\delta$, such that for $x_{i}>0$, for $i=1,2, \ldots, k+1$ and $u>0$,

$$
G\left(\sum_{i=1}^{k+1} x_{i}\right) \leq \delta \sum_{i=1}^{k+1} G\left(x_{i}\right), \quad G\left(u x_{i}\right) \leq G(u) G\left(x_{i}\right) ; \text { for each } i
$$

and that for $x_{i}<0$, for $i=1,2, \ldots, k+1$, and $u>0$,

$$
G\left(\sum_{i=1}^{k+1} x_{i}\right) \geq \delta\left(\sum_{i=1}^{k+1} G\left(x_{i}\right)\right), \quad G\left(u x_{i}\right) \geq G(u) G\left(x_{i}\right) ; \text { for each } i .
$$

Then every solution of (1.1) oscillates or tends to zero as $n \rightarrow \infty$.

Proof. By contradiction assume $y_{n}$ is an eventually positive solution of (1.1), which does not tend to zero as $n \rightarrow \infty$. Then there exists a $n_{0}$ such that for $n \geq n_{0}$ : $y_{n}, y_{\alpha(n)}, y_{\sigma(n)}$ are positive and $\lim \sup _{n \rightarrow \infty} y_{n}>0$. Define $c_{n}, z_{n}$ and $w_{n}$ by (2.8), (2.1) and (2.10) respectively to obtain (2.11). Then, $\Delta^{2} w_{n} \leq 0$ and $w_{n}, \Delta w_{n}$ are monotonic and of constant sign on some interval $\left[n_{1}, \infty\right)$. From $p_{n}^{\{j\}} \leq 0$ for each $j=1,2, \ldots, k$ and $y_{n}>0$, it follows that $w_{n} \geq y_{n}+c_{n}-F_{n}$. In the limit

$$
\lambda=\lim _{n \rightarrow \infty} w_{n} \geq \limsup _{n \rightarrow \infty} y_{n}>0 .
$$

Since $c_{n}$ and $F_{n}$ approach zero then $\lim _{n \rightarrow \infty} z_{n}=\lim _{n \rightarrow \infty} w_{n}=\lambda>0 . \quad z_{n}$ is bounded below by a positive constant, for all $n$ large. Using $y_{n}+\sum_{i=1}^{k} b_{i} y_{n-m_{i}} \geq$ $y_{n}-\sum_{i=1}^{k} p_{n}^{\{i\}} y_{n-m_{i}}, \lim _{n \rightarrow \infty} \sigma(n)=\infty$, and $\sigma\left(n-m_{j}\right)=\sigma(n)-m_{j}$, for eaach $j$, it follows that $y_{\sigma(n)}+\sum_{i=1}^{k} b_{i} y_{\sigma\left(n-m_{i}\right)}$ is also bounded below by a positive constant, on some interval $\left[n_{2}, \infty\right)$. Then by (G0)-(G1), there exist a positive constant $\beta$ such that $\beta \leq G\left(y_{\sigma(n)}+\sum_{i=1}^{k} b_{i} y_{\sigma\left(n-m_{i}\right)}\right)$. Using (2.18), we obtain

$$
\begin{aligned}
\beta & \leq G\left(y_{\sigma(n)}+\sum_{i=1}^{k} b_{i} y_{\sigma\left(n-m_{i}\right)}\right) \\
& \leq \delta\left[G\left(y_{\sigma(n)}\right)+\sum_{i=1}^{k} G\left(b_{i} y_{\sigma\left(n-m_{i}\right)}\right)\right] \\
& \leq \delta\left[G\left(y_{\sigma(n)}\right)+\sum_{i=1}^{k} G\left(b_{i}\right) G\left(y_{\sigma\left(n-m_{i}\right)}\right)\right] .
\end{aligned}
$$


From (2.11),it follows that

$$
\begin{aligned}
& \Delta^{2} w_{n}+\sum_{i=1}^{k} G\left(b_{i}\right) \Delta^{2} w_{n-m_{i}} \\
& \leq-v_{n}^{*}\left[G\left(y_{\sigma(n)}\right)+\sum_{i=1}^{k} G\left(b_{i}\right) G\left(y_{\sigma\left(n-m_{i}\right)}\right)\right] \\
& \leq-v_{n}^{*} \beta / \delta
\end{aligned}
$$

Taking summation from $n=n_{2}$ to $s-1$ we obtain

$$
\begin{aligned}
& \Delta w_{s}+\sum_{i=1}^{k} G\left(b_{i}\right) \Delta w_{s-m_{i}} \\
& \leq \Delta w_{n_{2}}+\sum_{i=1}^{k} G\left(b_{i}\right) \Delta w_{n_{2}-m_{i}}-(\beta / \delta) \sum_{r=n_{2}}^{s-1} v_{r}^{*} .
\end{aligned}
$$

In the limit as $s \rightarrow \infty$, by (2.17), the right-hand side approaches $-\infty$ while the left-hand side is positive. This contradiction proves that eventually positive solutions must converge to zero. For eventually negative solutions, one may proceed with substitution $x_{n}=-y_{n}>0$ and proceed as above for the case $y_{n}>0$ and complete the proof. Thus the proof is complete.

Remark 2.12. The above Theorem 2.11 generalizes and compliments [2][Theorem 3.6].

Remark 2.13. The condition (2.17) implies (G2). However, if $v_{n}$ is monotonic then both (2.17) and (G2) are equivalent. Indeed, if $v_{n}$ is decreasing then $v_{n}^{*}=v_{n}$. Hence the equivalence of (2.17) and (G2) is immediate. On the other hand if $v_{n}$ is increasing then assume that (G2) holds. As $v_{n}$ is increasing, $v_{n}^{*}=v_{n-r}$ where $r=\max \left\{m_{1}, m_{2}, \ldots, m_{k}\right\}$. Hence $\sum_{n=N_{1}}^{\infty} v_{n}^{*}=\sum_{n=N_{1}}^{\infty} v_{n-r}=\sum_{j=N_{1}+r}^{\infty} v_{j}=\infty$. Thus, (2.17) holds, and is equivalent to (G2), when $v_{n}$ is monotonic.

Theorem 2.14. Assume (G0)—(G6) to hold. Further, assume that $p_{n}^{\{j\}}, j=1,2, \ldots, k$ satisfy (2.16). Let $\sigma\left(n-m_{j}\right)=\sigma(n)-m_{j}$ for $j=1,2, \ldots, k$. Suppose that (2.18), (2.19) hold and that $v_{n}$ is monotonic. Then every solution of (1.1) oscillates or tends to zero as $n \rightarrow \infty$.

Proof. The proof follows from the proof of Theorem 2.11 and the remark 2.13.

Remark 2.15. The above result generalizes [2][Theorem 3.6]. 
Remark 2.16. The prototype of the function $G$ satisfying (G0), (G1), (2.18) and (2.19) is $G(u)=\left(\beta+|u|^{\mu}\right)|u|^{\lambda}$ sgnu, where $\lambda>0, \mu>0, \lambda+\mu \geq 1, \beta \geq 1$. For verification we may take help of the well known inequality(see[5, p.292])

$$
u^{p}+v^{p} \geq \begin{cases}(u+v)^{p}, & 0 \leq p<1, \\ 2^{1-p}(u+v)^{p}, & p \geq 1\end{cases}
$$

Theorem 2.17. Suppose that (G0),(G1), (G7) hold. Let $p_{n}^{\{j\}}, j=1,2, \ldots, k$ satisfy (2.5). Consider the homogeneous NDDE

$$
\Delta^{2}\left(y_{n}-\sum_{i=1}^{k} p_{n}^{\{i\}} y_{n-m_{i}}\right)+v_{n} G\left(y_{\sigma(n)}\right)=0 .
$$

Then the following are true.

(A) every bounded solution $y_{n}$ of (2.20) oscillates or tends to zero as $n \rightarrow \infty$.

(B) every unbounded solution $y_{n}$ of (2.20) oscillates or satisfies $\lim _{n \rightarrow \infty}\left|\sum_{i=1}^{k} y_{n-m_{i}}\right|=+\infty$ or $\liminf _{n \rightarrow \infty}\left|y_{n}\right|=0$.

Proof. Let $y_{n}$ be a eventually positive solution of (2.20). Then setting $z_{n}$ as in (2.1) we obtain

$$
\Delta^{2} z_{n}=-v_{n} G\left(y_{\sigma(n)}\right) \leq 0
$$

it follows that $z_{n}, \Delta z_{n}$ are monotonic and of constant sign on some interval $\left[n_{1}, \infty\right)$. By this we have two distinct possibilities; i.e, case (i) $\Delta z_{n}<0$ or case(ii): $\Delta z_{n}>0$.

Let us prove (A) and suppose that $y_{n}$ is bounded. As $p_{n}^{\{j\}}, j=1,2, \ldots, k$ satisfy (2.5), this implies $z_{n}$ is bounded. As $z_{n}$ is monotonic also then $\lambda:=\lim _{n \rightarrow \infty} z_{n}$ exists as a finite number. Let us consider case(i); i.e $\Delta z_{n}<0$. Then clearly, $\lim z_{n}=-\infty$, a contradiction. Then consider case(ii); i.e $\Delta z_{n}>0$. Then $\lim _{n \rightarrow \infty} \Delta z_{n}$ exists finitely. Summing (2.21) from $\mathrm{n}=n_{0}$ to $s-1$, and taking limit $s \rightarrow \infty$ we obtain

$$
\sum_{i=n_{0}}^{\infty} v_{i} G\left(y_{\sigma(i)}\right)<\infty
$$

Now we claim that $\lim \inf y_{n}=0$. Taking summation on (2.21), two times,

$$
z_{n}-\lambda=-\sum_{s=n}^{\infty}(s-n+1) v_{s} G\left(y_{\sigma(s)}\right) .
$$

Since $z_{n}$ is bounded, the above summation is convergent. This in turn, by (G7), implies $\liminf _{s \rightarrow \infty} G\left(y_{\sigma(s)}\right)=0$. As $G(x) \neq 0$ for $x \neq 0, \liminf _{s \rightarrow \infty} y_{\sigma(s)}=0$ 
and because $\lim _{n \rightarrow \infty} \sigma(n)=\infty, \liminf _{n \rightarrow \infty} y_{n}=0$. From lemma 2.1 it follows that $\lim _{n \rightarrow \infty} z_{n}=\delta=0$ and $\lim _{n \rightarrow \infty} y_{n}=0$. The proof for the case when $y_{n}$ is bounded and eventually negative solution of (2.20) is similar. Thus every bounded solution $y_{n}$ oscillates or tends to zero as $n \rightarrow \infty$ and part (A) of the theorem is proved. Let us prove (B) and suppose $y_{n}$ be an unbounded solution which is positive for $n \geq n_{0}$. First, case (i) may be considered. Consequently $\lim _{n \rightarrow \infty} z_{n}=-\infty$. As $p_{n}^{\{j\}}$ for $j=1,2, \ldots, k$ are bounded, then there exists a positive real $b$ such that $p_{n}^{\{j\}}<b$ for each $j$. Then

$$
\begin{aligned}
z_{n} & =y_{n}-\sum_{i=1}^{k} p_{n}^{\{i\}} y_{n-m_{i}} \\
& \geq-b \sum_{i=1}^{k} y_{n-m_{i}} .
\end{aligned}
$$

This implies $\sum_{i=1}^{k} y_{n-m_{i}} \geq \frac{z_{n}}{-b} \rightarrow+\infty$ as $n \rightarrow \infty$ So $\lim _{n \rightarrow \infty} \sum_{i=1}^{k} y_{n-m_{i}}=+\infty$. Consider case (ii). Then one may proceed as in the proof of part (A) and obtain $\liminf _{n \rightarrow \infty} y_{n}=0$. The proof for the case when $y_{n}$ is unbounded and eventually negative for large $n$ is similar. Thus the theorem is proved.

Theorem 2.18. Suppose that (G0),(G1),(G3)-(G6),(G8) hold. Let $p_{n}^{\{j\}}, j=1,2, \ldots, k$ satisfy

$$
0 \leq p_{n}^{\{j\}} \leq b, \quad \text { for } j=1,2, \ldots, k
$$

Then

(A) every bounded solution of (1.1) oscillates or tends to zero as $n \rightarrow \infty$.

(B) every unbounded solution $y_{n}$ of (1.1) oscillates or $\lim _{n \rightarrow \infty}\left|\sum_{i=1}^{k} y_{n-m_{i}}\right|=+\infty$.

Proof. Note that $(G 8)$ implies $(G 2)$. Let $y_{n}$ be a eventually positive solution of (1.1). Then setting $c_{n}, z_{n}$, and $w_{n}$ as in (2.8), (2.1) and (2.10), we obtain (2.11). From (2.11) , it follows that $w_{n}, \Delta w_{n}$ are monotonic and of constant sign. Then two distinct cases arise .i.e, case (i) $\Delta w_{n}<0$ or case (ii) $\Delta w_{n} \geq 0$. Let us prove (A). Suppose that $\left\{y_{n}\right\}$ is bounded and eventually positive solution of equation (1.1). The proof for the case when $y_{n}$ is eventually negative is similar. Then boundedness of $p_{n}^{\{j\}}, c_{n}, F_{n}$ implies $w_{n}$ is bounded. Consider the case (i) $\Delta w_{n}<0$, which implies that $\lim w_{n}=-\infty$, a contradiction due to the boundedness of $w_{n}$. Next consider case (ii) $\Delta w_{n}>0$ which yields

$$
\lim _{n \rightarrow \infty} \Delta w_{n}=l(\text { finite })
$$


Then summing (2.11) from $n=n_{1} \geq n_{0}$ to $s-1$, we obtain

$$
\Delta w_{n_{1}}-\Delta w_{s}=\sum_{n=n_{1}}^{s-1} v_{n} G\left(y_{\sigma(n)}\right) .
$$

Taking limit $s \rightarrow \infty$ in both sides of the above equation, one may obtain

$$
\sum_{j=n_{1}}^{\infty} v_{j} G\left(y_{\sigma(j)}\right)<\infty
$$

Then we claim that $\lim \sup _{n \rightarrow \infty} y_{n}=\beta=0$. If not, then $\beta>0$. Since $\lim _{n \rightarrow \infty} \sigma(n)=$ $\infty$ then $\lim _{\sup _{n \rightarrow \infty}} y_{\sigma(n)}=\beta$ and we find a subsequence $\left\{\sigma\left(n_{j}\right)\right\}$ of $\{\sigma(n)\}$ such that $\lim _{j \rightarrow \infty} y_{\sigma\left(n_{j}\right)}=\beta$. This implies that we can find a positive integer $n_{2}>n_{1}$ and a positive real $\eta$ such that $y_{\sigma\left(n_{j}\right)}>\eta>0$ for $j \geq n_{2}$. Then the sub sequence $y_{\sigma\left(n_{j}\right)}$ is bounded below by $\eta$ and above by $\beta+\epsilon$, where $\epsilon$ is arbitrary positive number. From continuity of $\mathrm{G}$ and (G0) we can find a positive lower bound $\delta$ for $G$ on $\{\eta, \beta+\epsilon\}$. Hence $G\left(y_{\sigma\left(n_{j}\right)}\right)>\mu>0$ for $j \geq n_{3} \geq n_{2}$

$$
\sum_{j=n_{3}}^{\infty} v_{n_{j}} G\left(y_{\sigma\left(n_{j}\right)}\right)>\mu \sum_{j=n_{3}}^{\infty} v_{n_{j}} \rightarrow \infty
$$

by (G8), which contradicts (2.24). Thus $\limsup _{n \rightarrow \infty} y_{n}=0$ which implies that $\lim _{n \rightarrow \infty} y_{n}=0$.

Let us next prove (B) and suppose $y_{n}$ be an unbounded positive solution of (1.1). We prove $\lim _{n \rightarrow \infty} \sum_{i=1}^{k} y_{n-m_{i}}=\infty$. The proof for the case when $y_{n}$ is unbounded and negative is similar. If case (i) happens then $\lim w_{n}=-\infty$ implies $\lim z_{n}=-\infty$. As $p_{n}^{\{j\}}$ for $j=1,2, \ldots, k$ are bounded, then there exists a positive real $b$ such that $p_{n}^{\{j\}}<b$ for each $j$. Then from (2.1), it follows that

$$
\begin{aligned}
z_{n} & =y_{n}-\sum_{i=1}^{k} p_{n}^{\{i\}} y_{n-m_{i}} \\
& \geq-b \sum_{i=1}^{k} y_{n-m_{i}} .
\end{aligned}
$$

This implies $\sum_{i=1}^{k} y_{n-m_{i}} \geq \frac{z_{n}}{-b} \rightarrow+\infty$ as $n \rightarrow \infty$

So $\lim _{n \rightarrow \infty} \sum_{i=1}^{k} y_{n-m_{i}}=+\infty$. If case(ii) happens i.e, $\Delta w_{n}>0$ then proceeding as before we arrive at (2.24). As $y_{n}$ is unbounded, there exists a subsequence $\left\{y_{\sigma\left(n_{j}\right)}\right\}$ of $y_{\sigma(n)}$ which $\rightarrow+\infty$ as $j \rightarrow \infty$. Hence $y_{\sigma\left(n_{j}\right)}>\gamma>0$ for $j \geq n_{2}$. Then by (G0), 
$G\left(y_{\sigma\left(n_{j}\right)}\right)>\eta>0$ for $n \geq n_{3} \geq n_{2}$. Therefore

$$
\sum_{j=n_{3}}^{\infty} v_{n_{j}} G\left(y_{\sigma\left(n_{j}\right)}\right) \geq \eta \sum_{j=n_{3}}^{\infty} v_{n_{j}} \rightarrow+\infty
$$

by (G8), a contradiction to (2.24). Thus case(ii) cannot happen. Then $\lim _{n \rightarrow \infty} \sum_{i=1}^{k} y_{n-m_{1}}=\infty$. Thus, the theorem is proved.

Remark 2.19. The above result generalizes [2][Theorem 3.12].

Next we present few examples to illustrate our results.

Example 2.20. Consider the higher order neutral difference equation with two delay terms

$$
\Delta^{2}\left[y_{n}+\left(2^{-n}+\frac{1}{16}\right) y_{n-4}+\left(2^{-n}+\frac{1}{32}\right) y_{n-5}\right]+\left[3\left(\frac{3}{4}\right)^{2} 2^{n-5}\right] y_{n-3}^{3}=f_{n}, \text { for } n>5
$$

where

$$
f_{n}=3\left(2^{-n-2}\right)+96\left(\frac{3}{4}\right)^{2} 2^{-2 n}
$$

Every non oscillatory bounded solution of (2.25) tends to zero as $n \rightarrow \infty$ by Theorem 2.5 or Theorem 2.7. As such $y_{n}=2^{-n}$ is a non oscillatory solution of (2.25) which tends to zero as $n \rightarrow \infty$.

Example 2.21. From Theorem 2.18 it follows that every unbounded solution of

$$
\Delta^{2}\left[y_{n}-\left(1+\frac{1}{2^{n}}\right) y_{n-1}-\left(4+\frac{1}{2^{n}}\right) y_{n-2}\right]+2^{\frac{2 n+1}{3}} y_{n-4}^{\frac{1}{3}}=0 .
$$

oscillates or tends to $\infty$ as $n \rightarrow \infty$. In particular, $y_{n}=2^{n}$ is the unbounded solution of the equation tending to $\infty$ as $n \rightarrow \infty$.

Example 2.22. Consider the neutral difference equation

$$
\Delta^{2}\left(y_{n}-p_{n}^{\{1\}} y_{n-1}-p_{n}^{\{2\}} y_{n-2}\right)+v_{n} y_{n-4}^{3}-2^{-n+1} H\left(y_{n-2}\right)=(-1)^{n-1} 2^{-n}, n \geq 4
$$

where

$$
\begin{array}{r}
v_{n}=-\frac{14}{3}, p_{n}^{\{1\}}=\frac{1}{2}-\frac{1}{n}, p_{n}^{\{2\}}=\frac{1}{3}-\frac{1}{n}, \\
u_{n}=2^{-n+1}, H(u)=\frac{u^{2}}{u^{2}+1} \operatorname{sgn}(\mathrm{u}) .
\end{array}
$$

Here $p_{n}^{\{j\}}$ satisfy (2.2) for $k=2$. From Theorem 2.5 and 2.8, it follows that every bounded solution (or solution) of (2.26) oscillates or tends to zero as $n \rightarrow \infty$. In partcular, (2.26) has a solution $y_{n}=(-1)^{n}$ which oscillates . 


\section{APPLICATION TO NEUTRAL DIFFERENCE EQUATIONS WITH OSCILLATING COEFFICIENTS}

In this section, we find sufficient conditions so that every solution of the higher order $(m \geq 2$ ) neutral differential equation (1.2) oscillates or tends to zero as $n \rightarrow \infty$, where $q_{n}$ is allowed to change sign. Let $q_{n}^{+}=\max \left\{q_{n}, 0\right\}$ and $q_{n}^{-}=\max \left\{-q_{n}, 0\right\}$. Then $q_{n}=q_{n}^{+}-q_{n}^{-}$and the equation (1.2) can be written as

$$
\Delta^{2}\left[y_{n}-\sum_{i=1}^{k} p_{n}^{\{i\}} y_{n-m_{i}}\right]+q_{n}^{+} G\left(y_{\sigma(n)}\right)-q_{n}^{-} G\left(y_{\sigma(n)}\right)=f_{n} .
$$

Now we proceed as in the previous section by setting $v_{n}=q_{n}^{+}, u_{n}=q_{n}^{-}$and $H(x)=G(x)$. Assumptions (G2), (G5), (G6) and (G7) become

(b2) $\sum_{n_{0}}^{\infty} q_{n}^{+}=\infty, \quad$ (b5) $\sum_{n_{0}}^{\infty} n q_{n}^{-}<\infty, \quad$ (b6) $\mathrm{G}$ is bounded,

(b7): $\sum_{n_{0}}^{\infty} n q_{n}^{+}=\infty$, respectively, which are feasible conditions. Therefore, the study of (1.2) reduces to the study of (1.1) in Theorems 2.5, 2.7, 2.8 and 2.11. Thus we can have the following results for (1.2), where $q_{n}$ changes sign.

Theorem 3.1. Suppose that $(\mathrm{G} 1)$, (G3),(G4),(b5) and (b7) hold. Assume that the sequence $\left\{p_{n}^{\{j\}}\right\}$ satisfies one of the four conditions (2.2)- (2.5). Then every bounded solution of (1.2) oscillates or tends to zero as $n \rightarrow \infty$.

Theorem 3.2. Assume (G0), (G1), (b2), (G3), (G4), (b5) and (b6)to hold. Further assume that $p_{n}^{\{j\}}$ satisfy (2.3). Then every solution of (1.2) oscillates or tends to zero as $n \rightarrow \infty$.

Theorem 3.3. Assume (G0), (G1),(b2), (G3), (G4), (b5), (b6) to hold. Suppose that $p_{n}^{\{j\}}$ lies in the range given by (2.2). Then every solution of (1.2) oscillates or tends to zero as $n \rightarrow \infty$.

Theorem 3.4. Assume (G0), (G1), (G3), (G4),(b5),(b6), (2.17), (2.18) and (2.19) to hold. Further, let $\sigma\left(n-m_{j}\right)=\sigma(n)-m_{j}$ for $j=1,2, \ldots, k$. Suppose that $p_{n}^{\{j\}}$ satisfy (2.16). Then every solution of (1.2) oscillates or tends to zero as $n \rightarrow \infty$.

For the results in this section, we need $G$ to be bounded, continuous, and to satisfy (G0) and (G1). The prototype of such a function is

$$
G(y)=y^{2 n} \operatorname{sgn}(y) /\left(1+y^{2 n}\right) .
$$

At the end, one example is presented to illustrate and signify our results. 
Example 3.5. Consider the non-linear neutral difference equation with several delay

$$
\Delta^{2}\left(y_{n}-\frac{1}{4} y_{n-1}-\frac{1}{8} y_{n-2}\right)+q_{n} G\left(y_{n-1}\right)=f_{n},
$$

where $G(u)=\frac{u^{2}}{\left(1+u^{2}\right)} \operatorname{sgn}(u)$. Let us define $q_{n}$ which changes sign, and $f_{n}$ as given below.

$$
\begin{gathered}
q_{n}= \begin{cases}1, & n \text { is odd, } \\
-2^{-n}, & n \text { is even. }\end{cases} \\
f_{n}= \begin{cases}4\left(2^{2 n}+4\right)^{-1}, & n \text { is odd, } \\
-4\left(2^{3 n}+2^{n+2}\right)^{-1}, & n \text { is even. }\end{cases} \\
\left\{q_{n}^{+}\right\}=\{1,0,1,0,1, \ldots,\} \text { and } q_{n}^{-}=\left\{0, \frac{1}{4}, 0, \frac{1}{16}, 0, \frac{1}{64}, \ldots\right\} .
\end{gathered}
$$

It is easily verified that eq. (3.2) satisfies all the conditions of Theorem 3.1 and 3.3. As such, every bounded solution of (3.2) oscillates or tends to zero as $n \rightarrow \infty$ and as such, this equation admits a non oscillatory solution given by $y_{n}=2^{-n}$ which tends to zero as $n \rightarrow \infty$. However, none of the results in the articles given in the reference can be applied to (3.2).

\section{CONCLUSION}

In this article, it is proved that the condition (b2) is sufficient under (b5) for every solution of (1.2)to be oscillatory or tending to zero, where $q_{n}$ changes sign. Note that the condition (b2) implies (G2), if (b5) holds. This could become a reality due to the relaxation of the condition that "For $|u|>0$ there exists $\delta>0$ such that $|G(u)| \geq \delta u$ "that was assumed in [10]. As a result, the left out class of sub linear NDDEs from [10] are included in this work and their oscillatory and asymptotic behavior are investigated. Further, some results for the unbounded solutions of (1.1) when $p_{n}^{\{j\}}$ satisfies (2.5) are obtained. Further investigation is needed for the behavior of unbounded solutions of (1.2) with $q_{n}$ changing sign when $G(x) \equiv x$, because in this case our assumption " $G$ is bounded" is not met. Let us have the following remark which would be helpful for a comparison of the results of this paper with that of [13].

Remark 4.1. As we assumed that the $p_{n}^{\{j\}}$, s are bounded sequences, therefore (2.16) is less restrictive than (2.4), and (2.23) is less restrictive than (2.5). If $p_{n}^{\{i\}}=p_{n}$ and $p_{n}^{\{j\}}=0$, for $j=1,2, \ldots, k$, but $j \neq i$, then the conditions (2.2), (2.3), (2.4), (2.5),(2.16) and (2.23) reduce to the conditions 
(A1): $0 \leq p_{n} \leq b<1, \quad(\mathrm{~A} 2):-1<-b \leq p_{n} \leq 0, \quad(\mathrm{~A} 3):-b_{1} \leq p_{n} \leq-b<-1$, (A4): $b_{1} \geq p_{n} \geq b>1, \quad(\mathrm{~A} 5):-\infty<-b \leq p_{n} \leq 0, \quad$ and (A6): $0 \leq p_{n} \leq b<\infty$, respectively. These conditions are assumed in $[8,9,13]$.

Note that, if $G$ is non decreasing then (G0) holds. Further, if $\sigma(n)=n-r$ then $\sigma(n-m)=\sigma(n)-m$. Moreover, if the condition $G(-u)=-G(u)$ holds then (2.18) implies (2.19). In view of these consideration and the above remark, some results of this work improve and generalize the following results of [13] as $G$ and $H$ are assumed to be non decreasing in [13].

Theorem 4.2. [13, Theorem 2.1]Suppose (A5) holds and $\sigma+1>m$. Assume (2.17) and (2.18) to hold and $G(-u)=-G(u)$ for $u>0$. Suppose that

$$
\int_{0}^{c} \frac{d u}{G(u)}<\infty
$$

Then every solution of (1.3) oscillates or tends to zero as $n \rightarrow \infty$.

Theorem 4.3. [13, Theorem 2.6] Suppose (A1) holds and $\sigma>m$. Assume the second condition of (2.18) to hold and $G(-u)=-G(u)$ for $u>0$. Suppose that (G2) and (4.1) hold. Then every solution of (1.3) oscillates or tends to zero as $n \rightarrow \infty$.

Theorem 4.4. [13, Theorem 2.10] Suppose (A4) holds and $m>2+\sigma$. Assume the second condition of (2.18) to hold and $G(-u)=-G(u)$ for $u>0$. Suppose that (G8) holds and

$$
\int_{c}^{\infty} \frac{d u}{G(u)}<\infty
$$

Then every solution of (1.3) oscillates or tends to zero as $n \rightarrow \infty$.

Theorem 4.5. [13, Theorem 2.9] Suppose (A4)and (G2) hold. Assume the second condition of (2.18) to hold and $G(-u)=-G(u)$ for $u>0$. Then every solution of (1.3) oscillates or tends to zero as $n \rightarrow \infty$.

The conditions " $G$ and $H$ are non decreasing "are relaxed in this work. As such, theorems $2.11,2.8$, and 2.18 of this work improve and generalize the theorems 4.2, 4.3 , and 4.4 respectively. Note that (G6) is not assumed in the results of [13]. But, with out (G6), the infinite series function $k(n)=\sum_{s=n}^{\infty}(s-n+1) r(s) H(y(s-\tau))$ defined in [13, page 253] is not well defined because, it cannot be proved that the infinite sum $k(n)$ is convergent under the assumption " $H$ is non decreasing"and a general $y_{n}$ which may be bounded or unbounded. This is a short coming of the paper [13]. Further, it seems Theorem 4.5 is a wrong resut as the neutral equation

$$
\Delta^{2}\left(y_{n}-4 y_{n-1}\right)+4^{(n+1) / 3} y_{n-2}^{1 / 3}=0
$$


satisfies all the conditions of Theorem 4.5, but, it admits a non oscillatory solution $y_{n}=2^{n}$, which tends to $\infty$, as $n \rightarrow \infty$, contradicting the theorem. Moreover, no result of the papers $[2,7,12,13,14]$ could be aplied to the examples 2.20, 2.21, 2.22 and 3.5, thus, signifying the importance of this work. Most importantly, theorems 3.1, 3.2, 3.3 and 3.4 of this article answers the open problem in [2, problem 4.2] for order 2. Before we close this article, we put an open problem before the readers for further research.

\section{PROBLEM}

We observe that, in all most all the results, concerned with the qualitative behaviour of inhomogeneous neutral difference equations of order $m \geq 1$, with positive and negative coefficients, the solutions oscillate or tend to zero as $n \rightarrow \infty$. Can stronger sufficient conditions be obtained so that all the solutions of these inhomogeneous or homogeneous neutral equations, would only oscillate.

\section{ACKNOWLEDGEMENT}

The authors are thankful to the referees for their valuable contributions for the better presentation of this article.

\section{REFERENCES}

[1] R. P. Agarwal, Difference Equations and Inequalities, Marcel Dekker, Newyork, 2000.

[2] Chittaranjan Behera;Radhanath Rath and Prayag Prasad Mishra Oscillation for second order neutral difference equations with variable delays. To appear in International J. of Mathematical, Engineering and Management Sciences, vol 5, n0. 4, (2020) pp. 663-681.

[3] L. H. Erbe, Q. K. Kong, B. G. Zhang; Oscillation Theory For Functional Differential Equations, Marcel Dekker, New York, 1995.

[4] I. Gyori and G. Ladas; Oscillation Theory of Delay-Differential Equations with Applications, Clarendon Press, Oxford, 1991.

[5] T. H. Hilderbrandt,Introduction to the Theory of Integration, Academic Press, New-York, 1963. 
[6] G. S. Ladde, V. Lakshmikantham and B. G. Zhang: Oscillation Theory of Differential Equations with Deviating Arguments, Marcel Dekker Inc.,New York, 1987.

[7] Karpuz, B., Ocalan, O., \& Yildiz, M. K.; Oscillation of a class of difference equations of second order, Math. Comput. Modelling, 49 (2009), 912-917.

[8] N. Parhi and A. K. Tripathy; Oscillation of forced nonlinear neutral delay difference equations of first order, Czech. Math. J., 53(2003), 83-101.

[9] R. N. Rath, B. L. S. Barik and S. K. Rath, Oscillation of higher order neutral Functional Difference Equations with positive and negative coefficients, Mathematica Slovaca Vol. 60, (2010) no.3, pp. 361-384.

[10] Radhanath Rath and Chittaranjan Behera; Oscillatory and asymptotic behaviour of a first order neutral equation of discrete type with variable several delay under $\Delta$ sign. International J. of Math. and Mathematical Sciences, Hindwai publishing House, Vol 2018, Artic;le ID 4586176, https//doi.org10.1155/20184586176

[11] H.L.Royden, "Real Analysis", MacMillan Publ. Co.(3rd .Edition),New York, 1989.

[12] E. Thandapani and K. Mahalingam,Necessary and sufficient conditions for oscillation of second order neutral difference equations, Tamkang J. Math., 34 (2003), 137-145.

[13] A. K. Tripathy, S. Panigrahi Oscillation in non linear neutral difference equations with positive and negative coefficients,Int. J. of Difference equations 5(2)(2010), 251-265.

[14] Yildiz, M.K., Karpuz , B. \& Ocalan, O.; Oscillation of nonlinear neutral delay differential equations of second-order with positive and negative coefficients,. Turkish J. Math., 33(4)(2009), 341-350.

[15] Yildiz, M. K., Oscillation of a class of nonlinear difference equations of second order with oscillating coefficients, Konuralp J. Math., 3(2)(2015), 211-218. 